
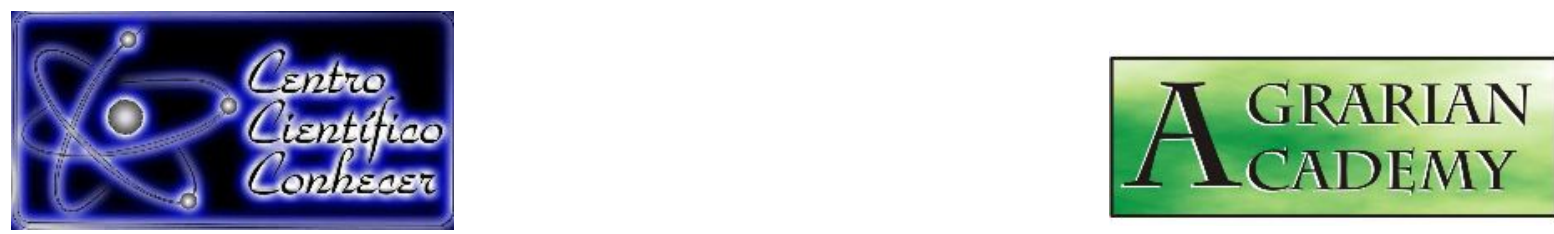

\title{
SIMULAÇÃO DE PERDA POR EVAPORAÇÃO COM DIFERENTES CALDAS E ADJUVANTES AGRÍCOLAS
}

\author{
Humberto Santiago', Tiago Mencaroni Guazzelli² \\ 1 Professor doutor da Universidade Federal do Oeste da Bahia, Barra-BA, Brasil. E-Mail; \\ humberto.ufob@gmail.com \\ 2 Estudante de doutorado do Departamento de Engenharia Agrícola da Universidade \\ Federal de Viçosa, Viçosa-MG, Brasil.E-mail; mencaroni.tiago@gmail.com \\ Recebido em: 15/12/2021 - Aprovado em: 15/12/2021 - Publicado em: 30/12/2021 \\ DOI: 10.18677/Agrarian_Academy_2021B7 \\ trabalho licenciado sob licença Creative Commons Attribution-NonCommercial-NoDerivatives 4.0 International License.
}

\begin{abstract}
RESUMO
A eficiência de uma aplicação de defensivos pode ser caracterizada em função das estratégias de controle que se adota, sendo uma delas a utilização de adjuvantes para melhorar o desempenho da calda de pulverização. Objetivou-se com o presente trabalho avaliar a evaporação de diferentes caldas contendo adjuvantes, em diferentes condições psicrométricas do ar. As caldas foram preparadas empregando-se seis adjuvantes e submetidas a cinco condições temperatura e umidade relativa diferente. Usou-se a massa das caldas contendo adjuvantes para mensurar a porcentagem de perda por evaporação. $O$ déficit de pressão de vapor de água no ar (DPVar) apresentou efeito significativo na massa evaporada das caldas avaliadas. Todas as soluções tiveram um aumento da porcentagem de perda por evaporação, de acordo com a drasticidade das condições climáticas. A maior porcentagem de perda por evaporação foi de $8,93 \%$ e foi constatada quando o déficit de pressão de vapor de água no ar alcançou o valor de $35 \mathrm{hPa}$.
\end{abstract}

PALAVRA CHAVE: agrotóxico, espectro de gotas e pulverização.

\section{SIMULATION OF EVAPORATION LOSS WITH DIFERENTE MISTURES AND AGRICULTURAL ADJUVANTS}

\begin{abstract}
The efficiency of a pesticide application can be characterized according to the control strategies that are adopted, one of which is the use of adjuvants to improve spray spray performance. The objective of this study was to evaluate the evaporation of different mixtures containing adjuvants, under different psychrometric conditions of the air. The solutions were prepared using six adjuvants and subjected to five different temperature and relative humidity conditions. The mass of the mixtures containing adjuvants was used to measure the percentage of loss by evaporation. The deficit of water vapor pressure in the air (DPVar) had a significant effect on the evaporated mass of the evaluated mixtures. All solutions had an increase in the percentage of loss by evaporation, according to the drasticity of climatic conditions. The highest percentage of evaporation loss was $8.93 \%$ and was found when the water vapor pressure deficit reached $35 \mathrm{hPa}$.
\end{abstract}

KEYWORDS: agrotoxic, drop spectrum and spraying 


\section{INTRODUÇ̃̃o}

O crescimento da produtividade agrícola é um dos fatores que asseguram a competitividade do setor. Diversas tecnologias são utilizadas com o objetivo de garantir a produção da planta, como o uso de agrotóxicos para controlar problemas fitossanitários. Estudos relacionados à qualidade da aplicação são fundamentais, uma vez que há diversos tipos de pulverizadores, produtos e formulações disponíveis no mercado (MARTINI et al., 2017).

A pulverização agrícola necessita de atenção, devido que no período da safra a pulverização de produtos fitossanitários ocorre até 40 vezes no mesmo ciclo da cultura. A aplicação de defensivos agrícolas apresentam perdas ente $40 \%$ a $60 \%$ em cada pulverização e $27 \%$ é considerada como evaporação, sendo esta perda antes e depois da gota chegar ao alvo. Ao analisar o volume e o valor financeiro que o Brasil utiliza de pesticida por ano, observa-se que em 2017 foi utilizado 539,9 mil toneladas com custo de US $\$ 8,8$ bilhões, entretanto, $27 \%$ do investimento em defensivos agrícolas é perdido, chegando US $\$ 2,37$ bilhões de dólares e 145,77 mil toneladas irão para o ar. (ANDEF, 2019).

O modelo de produção agrícola atual, a qualidade e a quantidade da produção dependem diretamente das pulverizações e por isso, quando essa atividade é realizada sem critérios técnicos, gera prejuízos sociais, ambientais e econômicos. A eficiência na aplicação de agrotóxicos é resultante de uma série de fatores, como seleção dos equipamentos, da calibração, das ferramentas e mecanismos que são capazes de reduzir as perdas por deriva, evaporação e escorrimento (PRECIPITO et al., 2018;RODRIGUES et al., 2019).

Como estratégia para aumentar a eficiência em uma pulverização geralmente são empregados os adjuvantes, diferentes pontas hidráulicas, redução do volume de calda entre outros, (CAIXETA et al., 2019). Antuniassi et al., (2013) definem adjuvante agrícola como qualquer substância acrescentada à calda de pulverização, sem contar os adjuvantes contidos na formulação do defensivo agrícola, que podem melhorar o seu desempenho na aplicação.

Algumas tecnologias têm sido pesquisadas e incorporadas às pulverizações agrícolas, visando auxiliar na aplicação correta de produtos fitossanitários (GITIRANA NETO et al., 2016; FRANÇA et al., 2017). Esses produtos alteram as características físico-químicas da calda, principalmente a viscosidade e tensão superficial (TOTOLI et al., 2016). Isso modifica, por consequência, o espectro de gotas geradas na pulverização, aumentando o diâmetro das gotas e reduzindo a deriva e evaporação. Porém, ao introduzir ferramentas e mecanismos em uma aplicação de agrotóxico devem-se levar em consideração as condições meteorológicas (umidade relativa do ar e temperatura) que podem gerar perdas consideravelmente elevadas durante a pulverização.

Assim, considerando a evaporação como a perda que ocorre pela vaporização das gotas durante o percurso da ponta até o alvo ou mesmo após a deposição no alvo devido ao déficit de pressão de vapor d'água no ar (DPVar), têmse como consequência, a redução da quantidade de ingrediente ativo que chega e é absorvido pelo o alvo. Dessa forma, o produto químico presente nas gotas pode contaminar o vapor d'água presente na atmosfera, podendo se transformar em água de chuva e, consequentemente, contaminar diferentes regiões rurais e urbanas (YU et al., 2009a; YU et al., 2009b).

As condições psicrométricas do ar (meteorológicas) são consideradas tão importantes que alguns estudos têm evidenciado que o tempo para a evaporação 
das gotas aumenta exponencialmente com o aumento da umidade relativa do ar, com registro de valores que chegam a 153\% quando se aumenta a UR de 30 para 90\% (YU et al., 2009a; YU et al., 2009b). Portanto, objetivou-se com esse trabalho avaliar a interação da superfície superior da calda pulverizada com 0 ar da atmosfera, entretanto será quantificada a perda por evaporação com diferentes caldas contendo adjuvantes, em diferentes condições psicrométricas do ar.

\section{MATERIAL E MÉTODOS}

O experimento foi realizado no Laboratório de Aplicação de Defensivos Agrícolas (LADA) do Departamento de Engenharia Agrícola, na Universidade Federal de Viçosa, campus de Viçosa-MG. Neste estudo, avaliou-se a massa de calda evaporada em 30 minutos com diferentes tipos de adjuvantes, levando-se em consideração a variação do déficit de pressão de vapor de água no ar e pressão de vapor do produto.

O trabalho foi conduzido no esquema fatorial de $6 \times 5$ (seis caldas $x$ cinco DPVar), com um total de 30 tratamentos (combinação dos fatores), utilizando-se 0 delineamento inteiramente casualizado (DIC), com três repetições por tratamento, totalizando 90 unidades experimentais. As amostras foram retiradas de soluções concentradas e as doses eram indicadas pelos fabricantes, onde os adjuvantes utilizados eram formulados a base de Resina Orgânica (R. O.) $\left(0,5 \mathrm{ml} \mathrm{L}^{-1}\right)$, Óleo Vegetal (O. V.) (1 L $300 \mathrm{~L}^{-1}$ de calda de pulverização), Óleo Mineral (O. M.) (5 ml L $\left.{ }^{1}\right)$, Silicone $\left(0,05\right.$ a $\left.0,1 \mathrm{ml} \mathrm{L}^{-1}\right)$, Ultra Baixo Volume Oleoso (UBVO) e água destilada (testemunha).

Foram retiradas, com o auxílio de uma pipeta, três alíquotas de $10 \mathrm{ml}$ de cada calda e depositadas em placas de Petri, com uma mesma superfície e área de contato. As placas foram pesadas antes e depois de receberem as amostras, em uma balança de precisão, marca AdventurerTM, modelo ARA520. Após as condições psicrométricas do ar estabilizadas, as placas de Petri foram colocadas sobre uma mesa de madeira com isolamento térmico no interior de uma câmara climática automatizada, hermeticamente fechada para impedir a entrada e saída de ar, possibilitando o controle da temperatura, a umidade relativa e a recirculação do ar.

A dimensão da câmara climática é de $3 \times 2 \times 2 \mathrm{~m}$ de comprimento, altura e largura respectivamente, construída com material de isolamento térmico, com paredes de $0,15 \mathrm{~m}$ de espessura em poliestireno, e revestida inteiramente com chapas galvanizadas de cor branca.

O monitoramento da temperatura e da umidade relativa do ar foi feito por meio de dois sistemas, um integrado ao painel de controle da câmara climática e outro instalado próximo das amostras. O painel de controle possui sistemas de controle de temperatura e umidade relativa do ar e capacidade de medição precisa por meio de um controlador lógico programável (CLP).

O sistema de monitoramento instalado era composto por um Thermohigrômetro modelo Ummi, marca Betha e o controle das condições climáticas da câmara automatizada era feito por sistemas de umidificação (marca Black \& Decker, modelo UC1000), aquecimento (marca Enxuta, modelo ST), desumidificação e resfriamento (ar condicionado marca Delta-Frio, modelo 9.000 BTU) e sistema de recirculação de ar composto por um ventilador axial modelo DF 15.15 do fabricante Delta-Frio com velocidade do ar de $1,45 \mathrm{~m} \mathrm{~s}^{-1}$.

Para alterar as condições de temperatura e umidade relativa do ar, foi feito o 
acionamento dos equipamentos que compunham o sistema descrito. O esquema da câmara climática e sua instrumentação podem ser observados na Figura 1.

FIGURA 1. Esquema da câmara climática utilizada no experimento

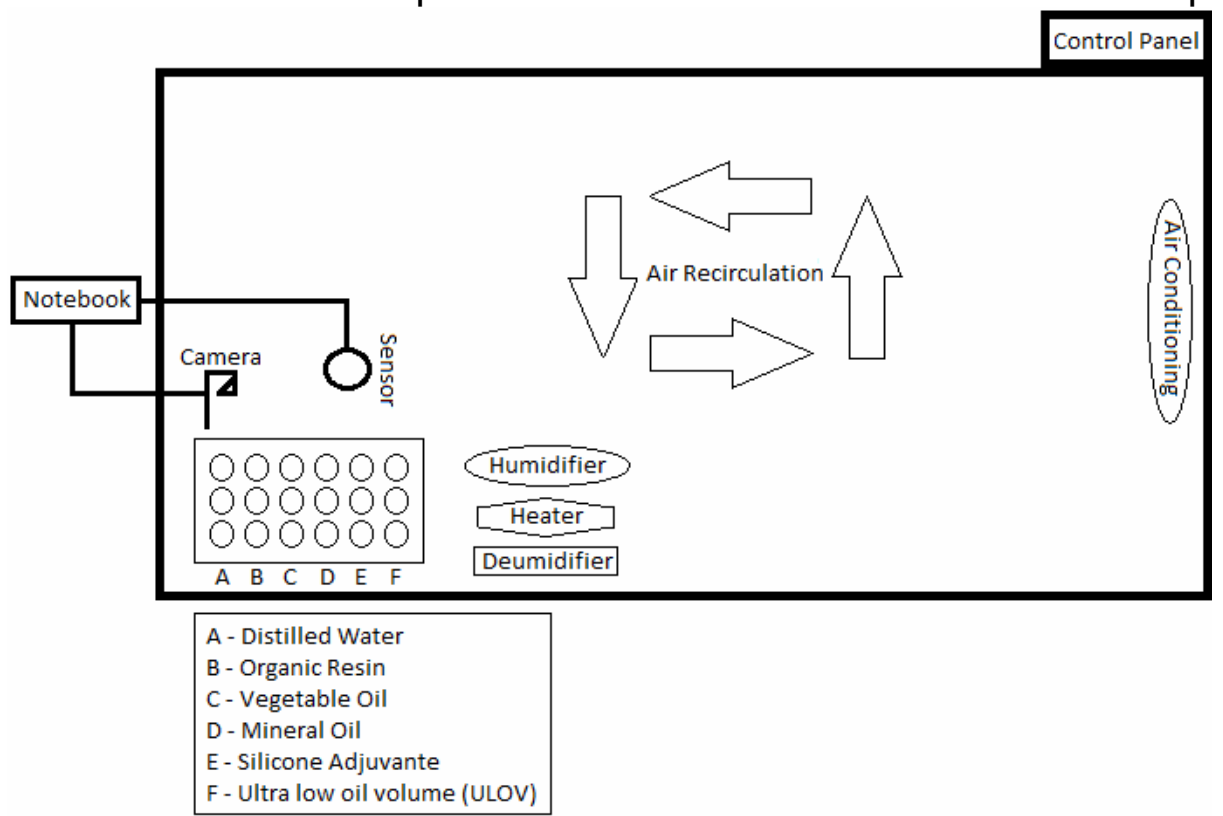

Fonte: Os autores (2021).

As amostras foram submetidas, na câmara climática, aos DPVar de 7, 14, 21, 28 e $35 \mathrm{hPa}$, e estes foram calculados, conforme a Equação 1.

$\mathrm{DPV}_{\mathrm{ar}}=\mathrm{e}_{\mathrm{s}}-\mathrm{e}_{\mathrm{a}}$

em que

DPVar = Déficit de Pressão de Vapor de Água no $\operatorname{Ar}(\mathrm{hPa})$;

$\mathrm{e}_{\mathrm{s}}=$ pressão parcial de vapor d' água (hPa); e,

$\mathrm{e}_{\mathrm{a}}=$ pressão de saturação de vapor d'água (hPa).

As amostras permaneceram na câmara climática por 30 minutos e para determinar o tempo de cada ensaio foi alocado no sistema um conjunto de filmagens composto por uma câmera digital de alta resolução FujiFilm modelo S3200 de 14 mega pixels e um notebook ASUS modelo SonicMaster. Ao serem retiradas, as amostras foram novamente pesadas, e as massas das caldas evaporadas foram calculadas subtraindo-se a massa da calda não evaporada da massa da calda evaporada, conforme as Equações 2, 3 e 4.

$$
m_{c}=m_{p+c}-m_{p}
$$

em que

$\mathrm{m}_{\mathrm{c}}=$ Massa da calda;

$\mathrm{m}_{\mathrm{p}+\mathrm{c}}=$ Massa da placa de Petri + calda; $\mathrm{e}$,

$\mathrm{m}_{\mathrm{p}}=$ Massa da placa de Petri;

$$
m_{c e}=m_{p+c e}-m_{p}
$$

em que

$m_{c e}=$ Massa da calda evaporada; 
$m_{p+c e}=$ Massa da placa de Petri + calda evaporada; ,

$\mathrm{m}_{\mathrm{p}}=$ Massa da placa de Petri;

$$
m_{e}=m_{c}-m_{c e}
$$

em que

$\mathrm{m}_{\mathrm{e}}=$ Massa evaporada $(\mathrm{g})$

$\mathrm{m}_{\mathrm{c}}=$ Massa da calda; $\mathrm{e}$,

$\mathrm{m}_{\mathrm{ce}}=$ Massa da calda evaporada .

Os dados seguiram a distribuição normal e foram submetidos à análise de variância, com significância de $1 \%$ e $5 \%$ de probabilidade pelo teste $F$, foi empregando-se teste de média TUKEY para comparar os adjuvantes com a água e utilizou o software SISVAR 3.0 para a regressão quadrática e o software SigmaPlot para desenvolvimento dos gráficos (porcentagem de massa evaporada x DPVar), para analisar o efeito do DPVar na diferentes caldas de pulverização

\section{RESULTADOS E DISCUSSÃO}

Os sistemas de monitoramento da temperatura e umidade relativa do ar não mostraram diferenças nas marcações e nas condições climáticas das mesmas, se mantendo estáveis. As estimativas das perdas por evaporação das caldas durante o período exposto às condições meteorológicas avaliadas seguem a normalidade dos dados e estão presentes na Figura 2. O DPVar apresentou efeito significativo na massa evaporada das caldas avaliadas.

FIGURA 2. Perdas por evaporação das soluções submetidas às condições climáticas.

$Y=-0,0058^{*} x^{2}+0,4106^{*} x+0,5487^{\text {ns }}$

$R^{2}=0,9106$

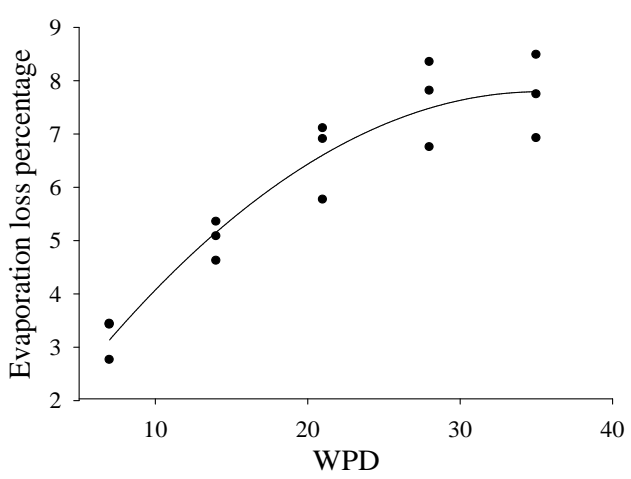

(a)

$$
\begin{aligned}
& Y=-0,0091^{* *} x^{2}+0,52^{*} x+0,5487^{n s} \\
& R^{2}=0,7607
\end{aligned}
$$

$Y=-0,004^{n s} x^{2}+0,3392^{* *} x+0,9849^{* *}$

$R^{2}=0,8120$

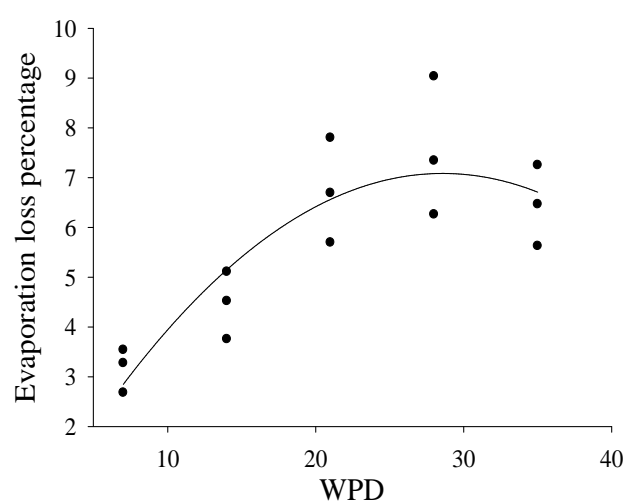

(b)

$Y=-0,0073^{* *} x^{2}+0,4823^{* *} x+0,1326^{* *}$

$R^{2}=0,8796$ 

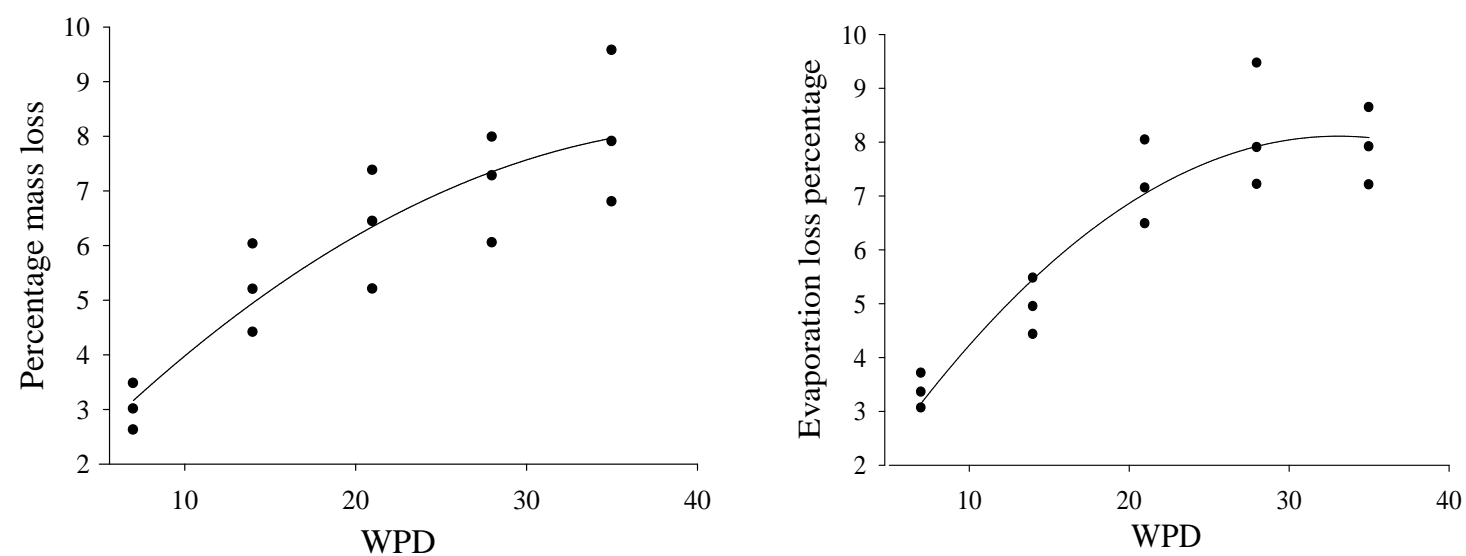

(c)

(d)

$Y=-0,0043^{n s} x^{2}+0,3781^{*} x+0,9697^{* *}$
$R^{2}=0,9104$

$Y=-0,0058^{n s} x^{2}+0,3139^{*} x+0,9849^{\text {ns }}$

$R^{2}=0,8120$

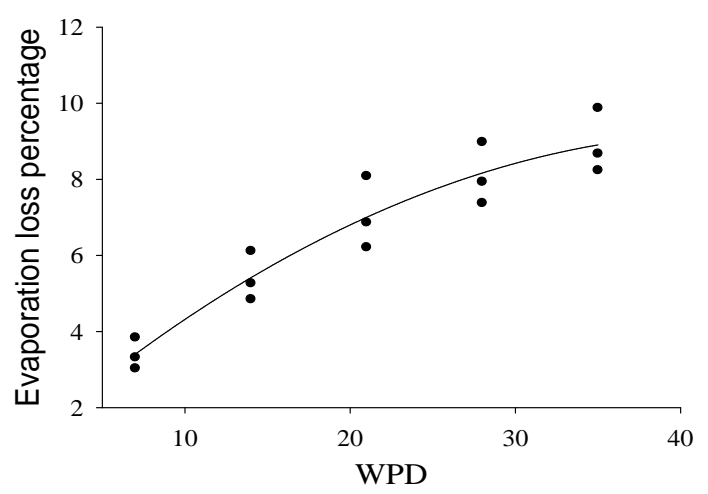

(e)

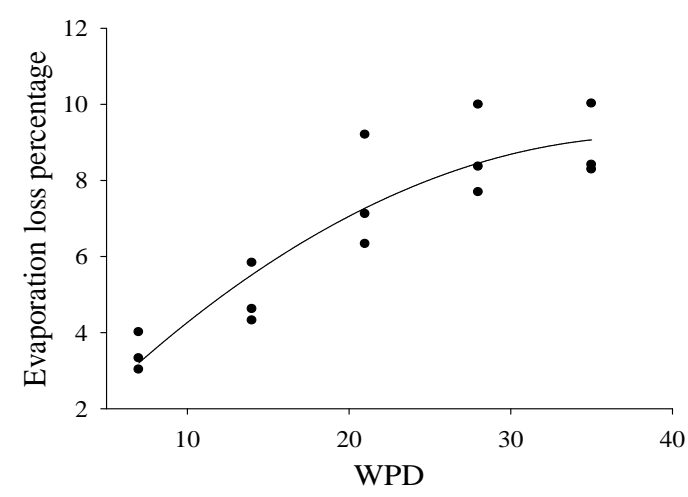

(f)

(a) Água Destilada; (b) Resina Orgânica; (c) Óleo Vegetal; (d) Óleo Mineral; (e) Siliconado; (f) UBVO. (significância a ** $1 \% \mathrm{e}^{*} 5 \%$ de probabilidade pelo teste $\mathrm{F}$ e ns - não significativo)

Todas as caldas tiveram um aumento da porcentagem de perda por evaporação, conforme as condições climáticas ficaram mais drásticas, evidenciando a importância de se realizar as aplicações de defensivos agrícolas em períodos do dia em que as condições climáticas estejam mais adequadas (início da manhã e fim da tarde). Relacionando com os estudos de pulverização de gota ao ar de diversos autores, observa-se que ao comparar diferentes horários de aplicação, constataram que a deposição do produto químico diminuiu significativamente, à medida que 0 déficit de pressão de vapor de água no ar é aumentado (BALAN et al., 2008; NASCIMENTO et al., 2012; ALVARENGA et al., 2013).

As perdas por evaporação durante a aplicação de agrotóxicos são consideradas uma das principais perdas que ocorrem nesta etapa da produção agrícola. Assim, para evitá-las, pode se utilizar produtos umectantes e que apresentem baixa pressão de vapor. A melhor forma de decidir uma aplicação será monitorando as condições psicrométricas do ar e não as tabelas de recomendações de temperatura do ar inferiores a $30{ }^{\circ} \mathrm{C}$ e de umidade relativa superiores a $55 \%$ (ANDEF, 2004; RAETANO, 2011). As porcentagens de perda por evaporação das caldas testadas, considerando os DPVar, podem ser observadas na Tabela 1. 
TABELA 1. Média da porcentagem de perda por evaporação das caldas em função dos DPVar.

\begin{tabular}{cccccc}
\hline \multicolumn{7}{c}{ DPVar $(\mathrm{hPa})$} \\
\hline & 7 & 14 & 21 & 28 & 35 \\
\hline Água & $3,21-\mathrm{c}$ & $5,02-\mathrm{d}$ & $6,59-\mathrm{b}$ & $7,64-\mathrm{c}$ & $7,72 \mathrm{~b}$ \\
R. O. & $3,17-\mathrm{b}$ & $\mathbf{4 , 4 6 - \mathrm { a }}$ & $6,73-\mathrm{c}$ & $7,55-\mathrm{b}$ & $\mathbf{6 , 4 5 a}$ \\
O. V. & $\mathbf{3 , 0 4 - a}$ & $5,21-\mathrm{e}$ & $\mathbf{6 , 3 4 - a}$ & $\mathbf{7 , 1 0 - a}$ & $8,09 \mathrm{~d}$ \\
O. M. & $3,38-\mathrm{d}$ & $4,95-\mathrm{c}$ & $7,22-\mathrm{e}$ & $8,19-\mathrm{d}$ & $7,92 \mathrm{c}$ \\
Silicone & $3,40-\mathrm{e}$ & $5,41-\mathrm{f}$ & $7,06-\mathrm{d}$ & $8,10-\mathrm{e}$ & $8,93 \mathrm{f}$ \\
UBVO & $3,46-\mathrm{f}$ & $4,92-\mathrm{b}$ & $7,55-\mathrm{f}$ & $8,68-\mathrm{f}$ & $8,91 \mathrm{e}$ \\
\hline
\end{tabular}

R.O. - resina orgânica; O.V. - óleo vegetal; O.M. óleo mineral; UBVO - ultra baixo volume oleoso. Letras minúsculas nas colunas diferenciam entre as médias.

Avaliando-se a porcentagem de perda por evaporação, quando se empregou - DPVar de $35 \mathrm{hPa}$, verificou-se que a perda foi de $7,72 \%$, para a água. Nas mesmas condições climáticas apenas o adjuvante formulado a base de resina orgânica reduziu a perda por evaporação para $6,45 \%$, sendo, uma redução na evaporação de $16,45 \%$ nas condições mais adversa da pulverização, entretanto, os demais adjuvantes apresentaram valores superiores ao da água. Este comportamento pode estar relacionado com o efeito umectante da formulação do produto, da tensão superficial (dependendo da interação molecular e de suas forças) e a viscosidade (relação de atrito interno entre as moléculas o qual reduz a agitação molecular), o adjuvante a base de óleo vegetal apresentou uma taxa de evaporação de $7,10 \%$, quando se empregou o DPVar de $28 \mathrm{hPa}$, sendo uma redução na evaporação de $7,06 \%$, e a mesma calda proporcionou no DPvar de 21 uma taxa de evaporação de 6,34 \%, sendo uma redução na evaporação de 3,79\%, ou seja, com o aumento da temperatura e a redução da umidade relativa do ar ocorreu um decréscimo da taxa de evaporação da calda. Portanto, segundo grandes grupos de fazendas e empresas de defensivos agrícolas na região de Luís Eduardo Magalhães 2020, se comparar a média de preço da aplicação para proteção de cultivo (crop protection) por área em lavoura de soja $(\$ 180,00 / \mathrm{ha})$ e algodão $(\$ 600,00 / \mathrm{ha})$, esta redução pode chegar para soja $\$ 29,61 /$ ha e para algodão $\$ 98,70 /$ ha.

Baio et al., (2015) avaliaram as alterações causadas por adjuvantes nas propriedades físico-químicas de caldas de pulverização sobre a área de molhamento de folhas de soja e sobre alteração do tamanho de gotas em um período de 30 minutos após a pulverização e verificaram que ao adicionar adjuvantes às caldas de aplicação, todos os adjuvantes Aller Biw, LI 700, Nimbus, TA 35 e Gota Max, com evaporação de 64,29, 66,52, 68,04, 68,64 e 68,16\%, respectivamente, apresentaram evaporação semelhante estatisticamente ao tratamento controle (água) 67,43\%, com exceção do adjuvante Break-Thru o qual ocasionou maior evaporação.

A água e as caldas a base de óleo vegetal, resina orgânica, silicone e UBVO apresentaram comportamento crescente na taxa de evaporação com o aumento do DPVar de 7 a $35 \mathrm{hPa}$. Desta forma o DPVar torna-se um parâmetro essencial na pulverização, pois ele relaciona as variáveis temperatura e umidade relativa do ar, que são fatores que devem ser monitorados concomitantemente durante uma aplicação de defensivos agrícolas. A contínua falta de monitoramento das condições psicrométricas (temperatura e umidade relativa do ar) pode acarretar perdas elevadíssimas, as quais podem alcançar valores de até $25 \%$ do total da calda aplicada segundo (BERG et al., 1999; CARLSEN et al., 2006a). Observou-se que ao 
adicionar adjuvantes ou produtos químicos que alterem as condições físicas e químicas da calda de pulverização, podem existir perdas ou não por evaporação em uma aplicação de defensivos agrícolas.

\section{CONCLUSÃO}

O déficit de pressão de vapor de água no ar é um parâmetro que influencia significativamente na evaporação de caldas de pulverização mesmo com a adição de adjuvantes independentemente da formulação.

O uso de adjuvantes ao decorrer do dia e nas horas de diferentes DPVar, deve ser direcionado a formulação ideal para minimizar o efeito da perda por evaporação.

Dependendo das propriedades físicas e químicas dos adjuvantes, a evaporação será maior ou menor, dependendo da pressão de vapor do produto e a quebra da tensão superficial respectivamente.

A R.O. e o O.V. foram os adjuvantes com menor influência de evaporação comparado com a água e os outros adjuvantes.

\section{AGRADECIMENTO}

Ao Departamento de Engenharia Agrícola da Universidade Federal de Viçosa, Capes e CNPQ pela concessão da bolsa de pesquisa.

\section{REFERÊNCIAS}

ALVARENGA, C. B.; TEIXEIRA, M. M.; CECON, P. R.; SIQUEIRA, D. L.; SASAKI, R. S.; RODRIGUES, D. E. Déficit de pressão de vapor d'água no ar na distribuição de líquido utilizando um pulverizador hidropneumático. Amazonian Journal of Agricultural and Environmental Sciences, Belém, v. 56, n. 2, p. 81-87, abr/jun. 2013.

ANTUNIASSI, U. R.; CHECHETTO, R. G.; MOTA, A. A. B.; CARVALHO, F. K.; SILVA, A.A.; VILELA, C. M. Influência de pontas de pulverização e adjuvantes no potencial de redução de deriva em túnel de vento. Semina, Londrina, v.34, n.1, p.3746, 2013.

ANDEF - Associação Nacional de defesa vegetal . Manual de Tecnologia de Aplicação de Produtos Fitossanitários. $1^{\underline{a}}$ edição, Campinas: Linea Creativa, 2004. 50 p.

ASSOCIAÇÃO NACIONAL DE DEFESA VEGETAL - ANDEF. Agrotóxico no Brasil: padrões de uso, política de regulação e prevenção da captura regulatória. Disponível em: Acesso em: 01/10/2019.

BAIO, F. H. R.; GABRIEL, R. R. F.; CAMOLESE, H. da S. Alteração das propriedades fisico-quimicas na aplicação contendo adjuvantes/changing the physical and chemical properties in the application containing adjuvants. Revista Brasileira de Engenharia de Biossistemas, v. 9, n. 2, p. 151-161, 2015.

BALAN, M. G.; ABI-SAAB, O. J. G.; SILVA, C. G.; RIO, A. Deposição da calda pulverizada por três pontas de pulverização sob diferentes condições 
meteorológicas. Semina: Ciências Agrárias, Londrina, v. 29, n. 2, p. 293-298, abr/jun. 2008.

BERG, F.; KUBIAK, R.; BENJEY, W. G.; MAJEWSKI, M. S.; YATES, S. R.; REEVES, G. L.; SMELT, J. H.; LINDEN, A. M. A. Emission of pesticides into the air. Water, Air and Soil Pollution, New York, v. 115, p. 195, 218, 1999.

CARLSEN, S. C. K. SPLIID, N. H.; SVENSMARK, B. Drift of 10 herbicides after tractor spray application. 1. Secondary drift (evaporation). Chemosphere, Philadelphia, v. 64, p. 787-794, 2006a.

CAIXETA, J. P. L.; FRANCO JÚNIOR, K. S.; BRIGANTE, G. P.; DIAS. M. S. Efeito de adjuvante associado a herbicidas no controle de digitaria insularis L. Revista Brasileira de Herbicidas, v. 18, n. 4. 2019.

FRANÇA, J. A. L.; CUNHA, J. P. A. R.; ANTUNIASSI, U. R. Spectrum, velocity and drift of droplets sprayed by nozzles with and without air induction and mineral oil. Engenharia Agrícola, v. 37, n. 3, p. 502-509, maio/jun. 2017. Disponível em: DOl:http://dx.doi.org/10.1590/1809-4430-Eng.Agric.v37n3p502-509/201

GITIRANA NETO, J.; CUNHA J. P. A. R.; MARQUES, R. S. ; LASMAR, O. ; BORGES, E. B. Deposição de calda promovida por pulverizadores empregados na cafeicultura de montanha. Coffee Science, v. 11, p. 267-275, 2016. Disponível em: http://www.sbicafe.ufv.br:80/handle/123456789/8071.

MARTINI, A. T.; SCHLOSSER, J. F.; BARBIERI, J. P.; BERTOLLO, G. M.; NEGRI, G. M.; BERTINATTO, R. Aspectos relevantes na inspeção de pulverizadores agrícolas: impactos na precisão das pulverizações de agrotóxicos. Acta Iguazu, Cascavel, v. 6, n. 4, p. 72-82, 2017.

NASCIMENTO, A. B.; OLIVEIRA, G. M.; BALAN, M. G.; HIGASHIBARA, L. R.; ABI-SAAB, O. J. G. Deposição de glifosato e utilização de adjuvante para diferentes pontas de pulverização e horário de aplicação. Pesquisa Aplicada \& Agrotecnologia, Guarapuava, v. 5, n. 2, p. 105-110, mai/ago. 2012.

PRECIPITO, L.M.B; DARIO, G.; OLIVEIRA, J.V.; OLIVEIRA, R.B.;Evaporation and wettability of fungicide spray, with or without adjuvant, on leaves of vegetables. Horticultura Brasileira, 36:p.320-324.2018. DOI - http://dx.doi.org/10.1590/S0102053620180306

RAETANO, C. G. Introdução ao estudo da tecnologia de aplicação de produtos fitossanitários. In: ANTUNIASSI, U. R.; BOLLER, W. (Org.). Tecnologia de aplicação para culturas anuais. Passa Fundo: Aldeia Norte; Botucatu: FEPAF, cap. 1, p. 15-26.2011.

RODRIGUES NETO, A. D.; ALMEIDA, M. S.; PRADO, E. P.; TROPALDI, L.; FIRMINO, A. C.; et al.; Otimização da atividade de herbicidas com diferentes adjuvantes na fitotoxidade de Digitaria insularis no estádio de florescimento. Colloquium Agrariae, v.15, n.5, p. 120-126, 2019. 
TOTOLI, D. S.; SOARES, J. P. C.; ALBERTON, O. Eficiência do grupo químico das carboxamida + estrobilurina no controle da ferrugem asiática em diferentes estádios da soja. Arquivos de Ciência Veterinárias e Zoologia da UNIPAR, v. 19, n. 3, p. 153- 157, 2016. Disponível em: https://www.revistas.unipar.br/index.php/veterinaria/ article/view/6088 DOI: http://dx.doi.org/10.25110/arqvet.v19i3.2016.6088

YU, Y.; ZHU, H.; FRANTZ, J. M.; REDING, M. E.; CHAN, K. C.; OZKAN, H. E. Evaporation and coverage area of pesticide droplets on hairy and waxy leaves. Biosystems Engineering, Philadelphia, v. 104, n. 3, p. 324-334, set. 2009a.

YU, Y.; ZHU, H.; OZKAN, H. E.; DERKSEN, R. C.; KRAUSE, C. R. Evaporation and deposition coverage area of droplets containing insecticides and spray additives on hydrophilic, hydrophobic, and crabapple leaf surfaces. Transactions of the ASABE, St. Joseph, v. 52, n. 1, p. 39-49, 2009b. 